\title{
Interplay between Depletion and Double-Layer Forces Acting between Charged Particles in Solutions of Like-Charged Polyelectrolytes
}

\author{
Mohsen Moazzami-Gudarzi, Tomislav Kremer, Valentina Valmacco, Plinio Maroni, Michal Borkovec, and Gregor Trefalt ${ }^{*}$ \\ Department of Inorganic and Analytical Chemistry, University of Geneva, \\ Sciences II, 30 Quai Ernest-Ansermet, 1205 Geneva, Switzerland
}

(Received 3 May 2016; published 15 August 2016)

\begin{abstract}
Direct force measurements between negatively charged silica particles in the presence of a like-charged strong polyelectrolyte were carried out with an atomic force microscope. The force profiles can be quantitatively interpreted as a superposition of depletion and double-layer forces. The depletion forces are modeled with a damped oscillatory profile, while the double-layer forces with the mean-field PoissonBoltzmann theory for a strongly asymmetric electrolyte, whereby an effective valence must be assigned to the polyelectrolyte. This effective valence is substantially smaller than the bare valence due to ion condensation effects. The unusual aspect of the electrical double layer in these systems is the exclusion of the like-charged polyelectrolyte from the vicinity of the surface, leading to a strongly nonexponential diffuse ionic layer that is dominated by counterions and has a well-defined thickness. As the oscillatory depletion force sets in right after this layer, this condition can be used to predict the phase of the oscillatory depletion force.
\end{abstract}

DOI: 10.1103/PhysRevLett.117.088001

Since the pioneering work of Asakura and Oosawa [1], depletion forces remained in the focus of the soft condensed matter community [2-4]. Much theoretical progress has been made by studying mixtures of hard spheres, and within the mean spherical approximation analytical expressions for the depletion potential between a pair of particles in a suspension of smaller depletants have been derived $[3,5]$. On the experimental side, spectacular results were obtained by exploring the possibility of tuning the range and strength of the interaction potential between colloidal particles through depletion forces induced by dissolved neutral polymers [6-8]. The availability of these systems opened the possibility to study their phase behavior and establish conditions concerning the occurrence of the gas-liquid phase transition [7] and its relation to colloidal aggregation [8].

More recently, the focus shifted towards charged depletants, including nanoparticles $[9,10]$, micelles $[11,12]$, or polyelectrolytes [13-15]. For a wide range of systems, these forces could be rationalized with a simple damped oscillatory profile, which follows from the large-distance asymptotics of the hard-sphere depletion potential $[3,9]$. The dependence of the free energy per unit area $W$ with separation distance $h$ as induced by the depletion interaction can be expressed as

$$
W_{\text {de }}(h)=A e^{-h / \xi} \cos (2 \pi h / \lambda+\theta),
$$

where $A$ is the amplitude, $\xi$ the correlation length, $\lambda$ the wavelength, and $\theta$ the phase shift. In contrast to hard-sphere systems, however, the wavelength shows a characteristic dependence on the number concentration $c$ of the depletants, typically with scaling behavior as $\lambda \propto c^{-\alpha}$, where $1 / 3 \leq \alpha \leq 1 / 2$. Recently, it was shown that this wavelength closely corresponds to the position of the structural peak observed in small angle scattering experiments [9].
When studying depletion forces, one always attempts to minimize interactions between the depletants and the respective substrate. For neutral polymers, the particles are often protected by alkyl-chain brushes [6]. For charged depletants, the larger particles are chosen to be highly charged and with a charge of the same sign as the one of the depletants $[9,11,14]$. In this way, the deposition of the depletants to the larger particles can be avoided. In such a system, however, the larger particles will interact by repulsive double-layer forces [2] and shorter-ranged depletion interactions [16]. Counterions may also alter the solvent structure close to the interface and affect the surface charge [17]. Double-layer forces will be intimately linked to depletion forces, since the charged depletants will contribute to screening. However, the combined action of the double-layer and depletion force was hardly studied. The reason could possibly be that the double-layer force does not decay exponentially, as one would naively expect from the simple theory of the electrical double layer.

Here, we investigate the interplay between the depletion and double-layer forces acting between charged colloidal silica particles in solutions of strong like-charged polyelectrolytes. We are able to quantify the forces measured with an atomic force microscope (AFM) by means of a superposition of the damped oscillatory depletion forces and repulsive double-layer forces. The double-layer force can be calculated accurately within the mean-field Poisson-Boltzmann (PB) theory by considering highly asymmetric electrolytes. Such electrolytes induce unusual nonexponential force profiles and a well-defined thickness of the diffuse part of the electrical double layer. This thickness then determines the onset of the depletion force and, in turn, its phase. 
The double-layer force between two equally charged plates is obtained by numerically solving the PB equation for constant charge boundary conditions [2]. For a solution containing different ions of number concentrations $c_{i}$ and valence $z_{i}$, the $\mathrm{PB}$ equation reads

$$
\frac{d^{2} \psi}{d x^{2}}=-\frac{q}{\varepsilon_{0} \varepsilon} \sum_{i} z_{i} c_{i} e^{-z_{i} q \psi / k T},
$$

where $q$ is the elementary charge, $\varepsilon_{0}$ the dielectric permittivity of vacuum, $\varepsilon$ the dielectric constant of water, $T$ the absolute temperature, and $k$ the Boltzmann constant. We use $T=298 \mathrm{~K}$ and $\varepsilon=80$ as appropriate for water at room temperature. From the electric potential at the midplane $\psi_{M}$, one obtains the double-layer disjoining pressure $\Pi_{\mathrm{dl}}$ given by [2]

$$
\Pi_{\mathrm{dl}}=k T \sum_{i} c_{i}\left(e^{-z_{i} q \psi_{M} / k T}-1\right),
$$

which is then integrated to obtain the interaction energy

$$
W_{\mathrm{dl}}=\int_{h}^{\infty} \Pi_{\mathrm{dl}}\left(h^{\prime}\right) d h^{\prime} .
$$

In the present situation of a highly asymmetric $1: Z$ electrolyte, where the multivalent coions have the same sign of charge as the surface, the pressure profile is determined by the salt-free situation. In this situation, the surface charge is only neutralized by the monovalent counterions, and the PB equation can be solved analytically. For large distances, the pressure is given by

$$
\Pi_{\mathrm{dl}}=\frac{\pi}{2} \frac{k T}{l_{B}\left(h+2 l_{\mathrm{GC}}\right)^{2}}-k T(1+Z) c,
$$

where $l_{B}=q^{2} /\left(4 \pi \varepsilon_{0} \varepsilon k T\right)$ is the Bjerrum length, $l_{\mathrm{GC}}=$ $2 \varepsilon_{0} \varepsilon k T /(q \sigma)$ is the Gouy-Chapman length, whereby $\sigma$ is the surface charge density, and $c$ is the number concentration of the $1: Z$ electrolyte. The second term corresponds to an osmotic correction [18]. Without that term and for $h \gg l_{\mathrm{GC}}$, Eq. (5) reduces to $\Pi_{\mathrm{dl}}=\pi k T /\left(2 l_{B} h^{2}\right)$ as initially proposed by Langmuir [19].

The numerically calculated PB pressure profiles are shown in Fig. 1. As the valence $Z$ of the coion is being increased, the profile becomes increasingly nonexponential [Fig. 1(a)]. A decrease of the salt concentration [Fig. 1(b)] has a similar effect. The limiting laws are also illustrated by comparing the exact PB profile with Eq. (5) and Langmuir's relation. The pressure decreases initially slowly but then decays rapidly due to the osmotic contribution in Eq. (5). This rapid decay leads to a well-defined thickness of the diffuse layer. The exponentially decaying DebyeHückel (DH) limiting law is recovered only at large distances [2]

$$
\Pi_{\mathrm{dl}}=2 \varepsilon_{0} \varepsilon \psi_{\mathrm{eff}}^{2} \kappa^{2} e^{-\kappa h},
$$
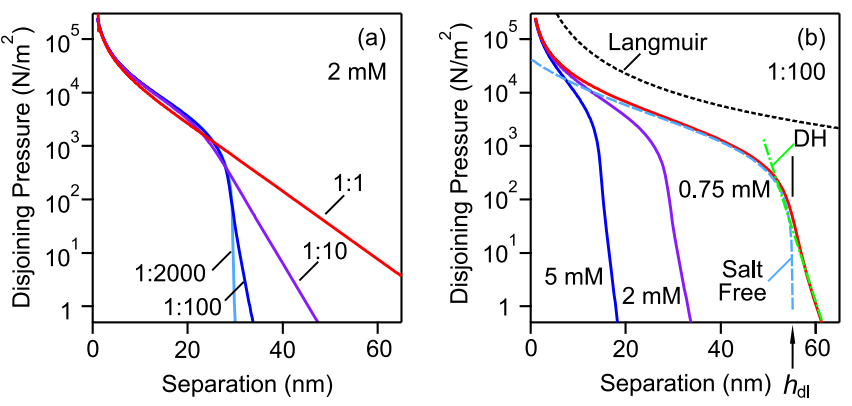

FIG. 1. Disjoining pressure $\Pi_{\mathrm{dl}}$ induced by the electrical double layer versus surface separation $h$ calculated numerically by solving the $\mathrm{PB}$ equation for highly asymmetric $1: Z$ electrolytes for a fixed surface change density of $-5 \mathrm{mC} / \mathrm{m}^{2}$ and different concentrations of monovalent ions. Influence of (a) valence $Z$ at a concentration of $2 \mathrm{mM}$ and (b) for a 1:100 electrolyte of different concentrations. The lowest concentration in (b) also indicates the Langmuir's relationship, approximate salt-free relation Eq. (5), DH limiting law Eq. (6), and the thickness of the diffuse layer $h_{\mathrm{d} l}$. The concentration indicated corresponds to the monovalent counterion concentration.

where $\psi_{\text {eff }}$ is the effective potential and $\kappa$ is the inverse Debye length defined by the relation $\kappa^{2}=4 \pi l_{B} \sum_{i} z_{i}^{2} c_{i}$. For such highly asymmetric electrolytes, the respective effective potentials become huge, which simply reflects the fact that the DH limiting law sets in only for $h \gg \kappa^{-1}$. Therefore, the DH approximation cannot be used [10], and the consideration of the PB theory becomes essential.

Very similar features can be observed experimentally. Interaction forces between two silica particles were measured with the colloidal probe technique [20,21]. This technique was implemented with a closed-loop AFM (MFP-3D, Oxford Instruments) mounted on an inverted optical microscope (Olympus, IX 70). Monodisperse silica particles (Bangs Laboratories) were placed to a quartz substrate and glued (Araldite 2000+) to a tipless AFM cantilever (Micromash, CSC37). The particles were heat treated at $1150{ }^{\circ} \mathrm{C}$ during $3 \mathrm{~h}$, which results in a solid attachment to the substrate and the cantilever. The heattreated particles were cleaned in air plasma (PDC-32 G, Harrick) and subsequently washed with ethanol and Milli-Q water (Millipore). The average particle radius is $2.20 \mu \mathrm{m}$ with a coefficient of variation of $1.2 \%$ measured with scanning electron microscopy. The root mean square (rms) roughness of $0.81 \pm 0.09 \mathrm{~nm}$ was determined by AFM imaging [22]. The attached particles were mounted in the AFM fluid cell, which was filled with a solution of sodium poly(styrene sulfonate) (NaPSS) (Polymer Standards, polydispersity index $<1.2$ ) adjusted to $p \mathrm{H} 4.0$ with $\mathrm{HCl}$. The particles were centered by means of the optical microscope with a precision of $\sim 50 \mathrm{~nm}$. Force profiles were extracted from the approach parts of vertical approach-retraction cycles. The contact point was determined from the onset of the constant compliance region with an accuracy of $\sim 0.5 \mathrm{~nm}$. The spring constants 

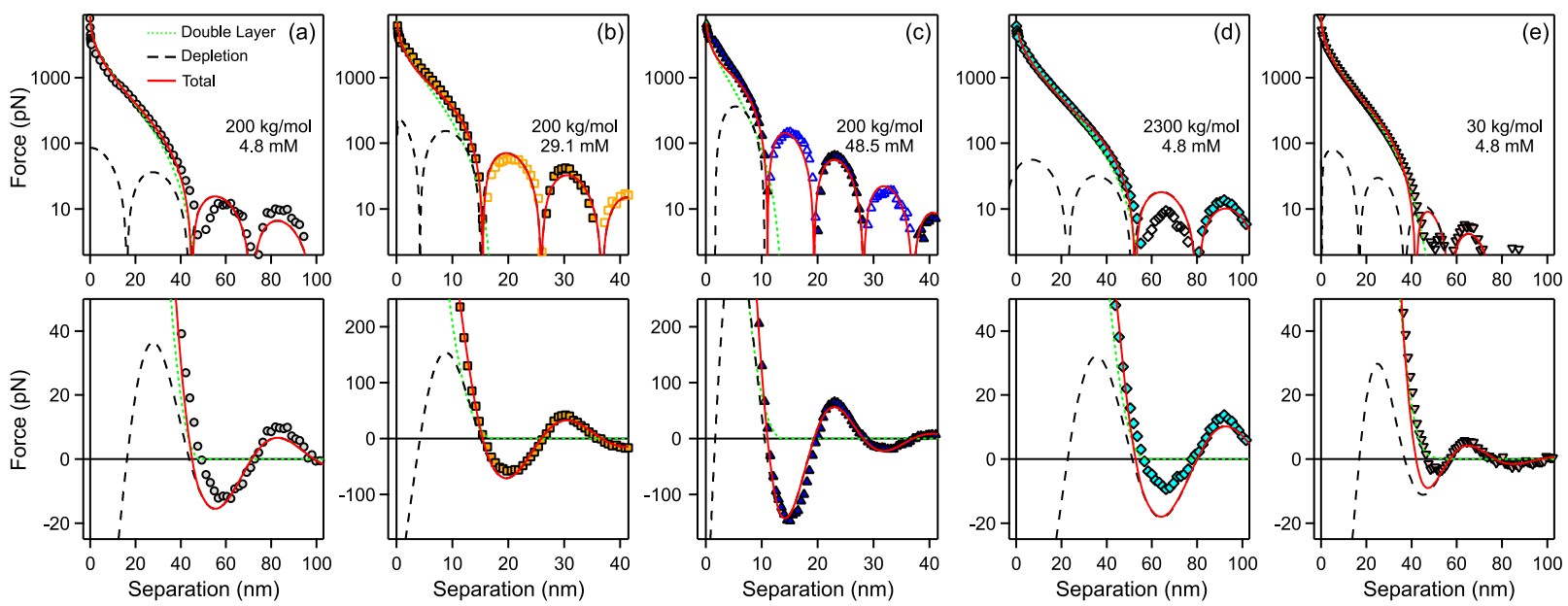

FIG. 2. Forces between silica particles in solutions of NaPSS. Experimental data are compared with calculations based on the PB theory for asymmetric electrolytes and a damped oscillatory depletion force. The concentration indicated corresponds to the monomer concentration. The semilogarithmic representations plot the magnitude of the force. (a)-(c) Variation in concentration and (a),(d),(e) in molecular mass.

of the cantilevers were $\sim 0.3 \mathrm{~N} / \mathrm{m}$ as determined by the thermal fluctuation method. By a subsequent averaging of about 100 force profiles, we obtain a force resolution of $\sim 2 \mathrm{pN}$. Force profiles obtained from the approach and retraction traces agreed well for cantilever velocities $<0.5 \mu \mathrm{m} / \mathrm{s}$. The force profiles measured between different pairs of particles were well reproducible at larger distances, while at shorter distances a variation of about $30 \%$ was observed. These observations confirm the symmetry of the present measurement geometry, while the lack of this symmetry was considered as a problem in a similar study [10]. Measurements were done also in dialyzed NaPSS solutions, but they were less well reproducible. These measurements showed that a minor changes in the $p \mathrm{H}$ and background solution concentration yields very similar results. We have further verified that PSS adsorption on silica is negligible. The fraction of molecular pulling events in the AFM force experiments was $<1 \%$ for all PSS samples with molecular mass $>30 \mathrm{~kg} / \mathrm{mol}$. PSS adsorption was also measured on oxidized silicon wafers by optical reflectivity with a home-built reflectometer. This fixedangle reflectometer involves a frequency-modulated polarized diode laser with a wavelength of $533 \mathrm{~nm}$, and an incidence angle of $60^{\circ}$ was used [23]. The adsorbed mass of PSS was $<50 \mu \mathrm{g} / \mathrm{m}^{2}$ in the relevant concentration range.

The force profiles shown in Fig. 2 were quantified by invoking the Derjaguin approximation, which states that the force is given by $F=\pi R W$, where $R$ is the mean particle radius and $W$ is the interaction energy [2]. We approximate this quantity by superposing the contributions from double-layer and depletion forces, namely,

$$
W=W_{\mathrm{dl}}+W_{\mathrm{de}} .
$$

The depletion contribution is given by Eq. (1), while the double-layer interaction was calculated with the full PB equation assuming a mixture of $0.1 \mathrm{mM}$ monovalent electrolyte and the appropriate concentration of $1: Z$ electrolyte. Such results were fitted to the measured force profiles. Thereby, the monomer concentration and the surface charge density $\sigma$ were fixed. The former was known from the analytical PSS concentration, while the latter value of $-5.0 \mathrm{mC} / \mathrm{m}^{2}$ was determined by direct force measurements in $10 \mathrm{mM} \mathrm{NaCl}$ solution adjusted to $p \mathrm{H} 4.0$. The remaining fitting parameters include the ones of the depletion force, namely, its amplitude $A$, the correlation length $\xi$, the wavelength $\lambda$, and the phase shift $0 \leq \theta<2 \pi$, and the only free parameter entering the double-layer force is the valence. No satisfactory fits could be obtained with the bare valence $Z$ of PSS, which corresponds to the number of charged groups. Therefore, we have introduced an effective valence $Z_{\text {eff }}$ as a fitting parameter. The fits systematically yields $Z_{\text {eff }}<Z$, and this difference implies that not all counterions dissociate. This phenomenon is well known as the Manning counterion condensation [24]. The experimental data were compatible with a fixed ratio $\lambda / \xi$ for each molecular mass. As Fig. 2 illustrates, this model was capable to quantify the experimental force profile very well, typically over 3 orders in magnitude. The resulting parameters are summarized in Figs. 3 and 4. The limiting DH decay is hardly noticeable in the force profiles, as it is hidden by the onset of the depletion force. Van der Waals attraction was not observed either, as it is probably overruled by short-ranged repulsive forces (e.g., hydration and hairy-layer) [20].

Figure 2 illustrates that double-layer forces dominate the force profiles at smaller distances, while depletion forces at larger ones. Because of the rapid decay of the former, both forces contribute simultaneously only in a small distance range. In this range, some deviations from the superposition approximation [Eq. (7)] can be evidenced. However, these deviations are relatively minor, and for this reason we did 

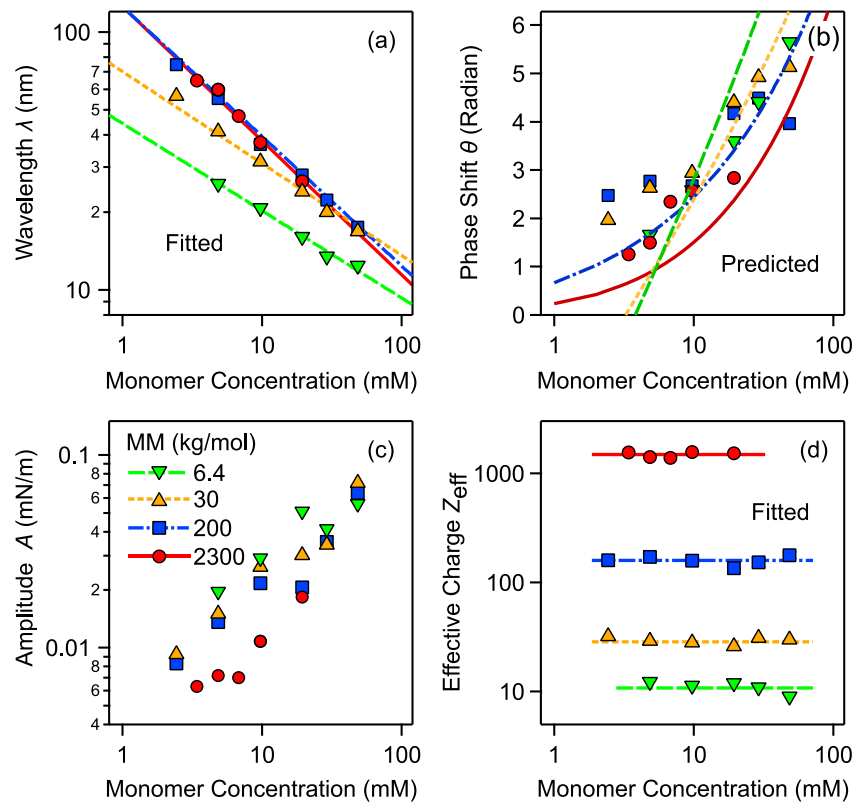

FIG. 3. Fitted parameters extracted from the measured force profiles versus the monomer concentration for NaPSS of different molecular mass (MM). (a) Wavelength $\lambda$, (b) phase shift $\theta$, (c) amplitude $A$, and (d) effective valence $Z_{\text {eff }}$. The solid lines indicate the fitted exponent in the power-law dependence $\lambda \propto c^{-\alpha}$ in (a) and the average value in (d). The solid lines in (b) are model predictions as described in the text.

not attempt to use more detailed models of the depletion force [3,5]. At shorter distances, the depletion force is overwhelmed by the double-layer force by orders of magnitude.

One further observes that the range of the double-layer force increases with increasing molecular mass of the polymer and decreases with increasing concentration. These trends were already suggested by the model calculations with the strongly asymmetric electrolyte presented in Fig. 1. The wavelength of the depletion force also increases with increasing molecular mass of the polymer and decreases with increasing concentration. Such trends were reported by researchers focusing on forces induced by charged depletants earlier $[13,25,26]$.

The dependence of the fitted parameters on the PSS concentration is summarized in Fig. 3. Let us first discuss the parameters describing the depletion force, namely, the wavelength $\lambda$, the phase shift $\theta$, and the amplitude $A$. The wavelength decreases with the concentration following a power law $\lambda \propto c^{-\alpha}$. The phase shift $\theta$ and the amplitude $A$ increase with concentration. Figure 4 summarizes the molecular mass dependence of the ratio $\lambda / \xi$ and the power-law exponent $\alpha$, and they compare favorably with values obtained from earlier direct force measurements in similar systems $[13,25,26]$. The fixed ratio $\lambda / \xi$ decreases with the molecular mass. The power-law exponent $\alpha$ increases from $1 / 3$ to $1 / 2$ at a molecular mass of $\sim 100 \mathrm{~kg} / \mathrm{mol}$ as the solution undergoes a dilute to

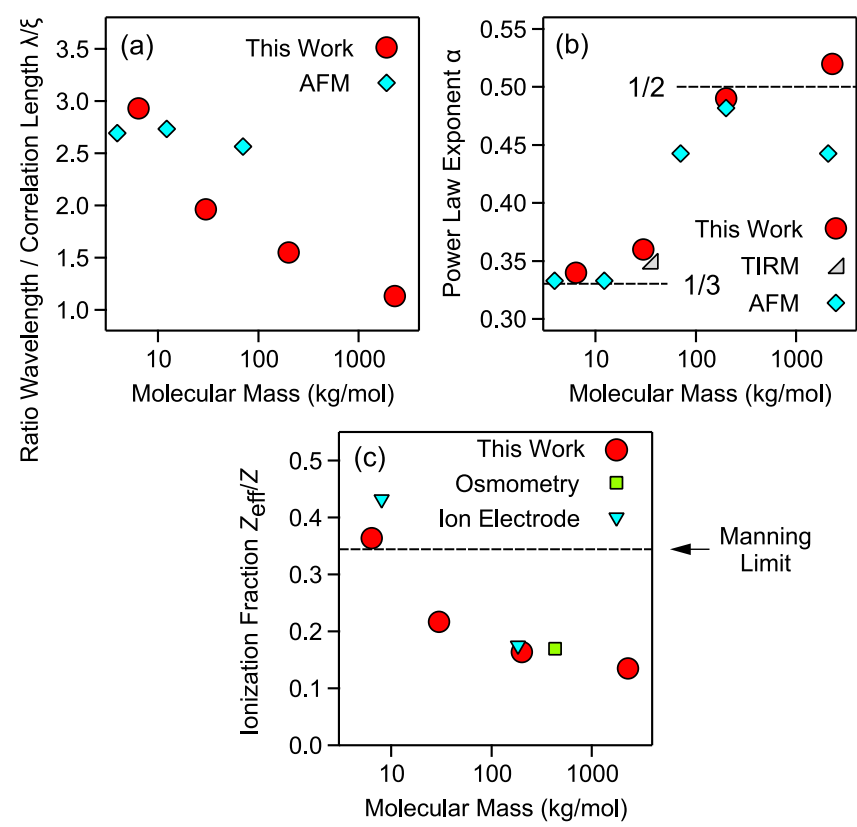

FIG. 4. Dependence of the model parameters in salt-free NaPSS solutions versus the molecular mass. (a) Ratio of the wavelength and correlation length $\lambda / \xi$, (b) exponent of the power law $\lambda \propto c^{-\alpha}$, and (c) ionization fraction $Z_{\text {eff }} / Z$. The present data are compared with parameters given in the literature for measured depletion forces in the same system for AFM colloidal probe measurements [13,25] and TIRM [26]. Literature data for the ionization fraction were obtained by osmometry [27] and ionspecific electrodes [28]. The dashed lines indicate the limiting exponents in (b) and the Manning limit in (c).

semidilute transition. For this molecular mass, the crossover concentration is about $\sim 7 \mathrm{mM}$ as can be inferred from the respective gyration radii [29].

The only adjustable parameter characterizing the doublelayer force is the effective valence $Z_{\text {eff }}$. This parameter is always lower than the bare valence $Z$, since some of the counterions are condensed on the polymer and they do not dissociate [24]. The effective valence is concentration independent [Fig. 3(d)] but increases with an increasing molecular mass of PSS. The ratio $Z_{\text {eff }} / Z$ decreases initially with the molecular mass but then remains almost constant around $0.15 \pm 0.05$ [Fig. 4(c)]. The presently measured values are very similar to values found with independent methods earlier [27,28]. The measured values are well comparable to the Manning condensation limit of $Z_{\text {eff }} / Z=a / l_{B} \simeq 0.35$, where $a \simeq 0.25 \mathrm{~nm}$ is the length of the PSS monomeric unit [24]. The experimentally observed values are somewhat smaller, and this deviation is probably caused by the small amounts of salt present [30]. Indeed, only a minor fraction of the counterions dissociated from the polyelectrolytes contribute to the diffuse layer formed.

This quantitative picture can be used to explore the interplay between depletion and double-layer forces. We now demonstrate that the phase of the depletion force is 
determined by the thickness of the diffuse layer. This thickness $h_{\mathrm{dl}}$ can be estimated by setting $\Pi_{\mathrm{dl}}\left(h_{\mathrm{dl}}\right)=0$ in the approximate Eq. (5) as indicated in Fig. 1(b). Once this distance is known, one requires that the argument in Eq. (1) is $2 \pi h_{\mathrm{dl}} / \lambda+\theta=5 \pi / 2$. The physical meaning of this condition is that the depletion force vanishes at the diffuse layer boundary and initially decreases with the distance. A similar condition is approximately valid for hard spheres [3]. From this condition, the phase $\theta$ can be calculated, whereby the wavelength $\lambda$ is obtained from the fitted scaling relations shown in Fig. 3(a). These predictions do not contain any adjustable parameters, and they are plotted as lines in Fig. 3(b). The agreement with the experiment is very good. This finding demonstrates that the onset of the depletion force is related to the thickness of the diffuse part of a polyelectrolyte-free electric double layer.

We conclude by stating that such a consistent description of forces acting on charged surfaces in solutions of likecharged polyelectrolytes by means of a superposition of depletion and double-layer forces has been proposed for the first time. The oscillatory depletion forces dominate at larger distances, whereby the respective wavelength reflects the structure of the polyelectrolyte solution and is in agreement with previous studies. The polyelectrolyte is excluded from the diffuse part of the double layer, and therefore this layer is dominated by monovalent counterions. This structure leads to a highly nonexponential force profile, which can be well rationalized by the PB theory for highly asymmetric electrolytes with monovalent counterions and multivalent polyelectrolyte coions. However, an effective valence must be assigned to the polyelectrolyte, which is typically $2-8$ times smaller than its bare value. The effective valence is comparable to the one obtained with independent methods [27,28] and can be relatively well rationalized by counterion condensation [24,30]. The thickness of this diffuse layer further determines the onset of the depletion force and its phase. While a DH regime is also present at large distances and low forces, this regime is typically hidden by the onset of the depletion force.

The present findings will certainly revive experimental and theoretical investigations of the structure of the double layer in strongly asymmetric electrolytes, which include not only polyelectrolyte solutions but also micellar solutions or salt-free suspensions of charged colloidal particles [9-12]. Similar investigations in the latter systems may also shed light on like-charge attraction phenomena [31], which might be related to the interplay of nonexponential force profiles originating from overlapping double layers and oscillatory depletion forces.

This research was supported by the Swiss National Science Foundation (Grant No. 159874) and the University of Geneva.
* Corresponding author. gregor.trefalt@unige.ch

[1] S. Asakura and F. Oosawa, J. Chem. Phys. 22, 1255 (1954).

[2] W. B. Russel, D. A. Saville, and W. R. Schowalter, Colloidal Dispersions (Cambridge University Press, Cambridge, England, 1989).

[3] A. Trokhymchuk, D. Henderson, A. Nikolov, and D. T. Wasan, Langmuir 17, 4940 (2001).

[4] K. Binder, P. Virnau, and A. Statt, J. Chem. Phys. 141, 140901 (2014).

[5] D. Henderson and K.-Y. Chan, Mol. Phys. 98, 1005 (2000).

[6] A. Milling and S. Biggs, J. Colloid Interface Sci. 170, 604 (1995).

[7] H. N. W. Lekkerkerker, W. C. K. Poon, P. N. Pusey, A. Stroobants, and P. B. Warren, Europhys. Lett. 20, 559 (1992).

[8] P. J. Lu, E. Zaccarelli, F. Ciulla, A. B. Schofield, F. Sciortino, and D. A. Weitz, Nature (London) 453, 499 (2008).

[9] S. H. L. Klapp, Y. Zeng, D. Qu, and R. von Klitzing, Phys. Rev. Lett. 100, 118303 (2008).

[10] S. Grandner, Y. Zeng, R. von Klitzing, and S. H. L. Klapp, J. Chem. Phys. 131, 154702 (2009).

[11] P. Richetti and P. Kekicheff, Phys. Rev. Lett. 68, 1951 (1992).

[12] D. 1. Sober and J. Y. Walz, Langmuir 11, 2352 (1995).

[13] C. Uzum, S. Christau, and R. von Klitzing, Macromolecules 44, 7782 (2011).

[14] S. Biggs, J. L. Burns, Y. D. Yan, G. J. Jameson, and P. Jenkins, Langmuir 16, 9242 (2000).

[15] A. J. Milling and K. Kendall, Langmuir 16, 5106 (2000).

[16] M. M. Hatlo, R. A. Curtis, and L. Lue, J. Chem. Phys. 128, 164717 (2008).

[17] S. Deshmukh, G. Kamath, S. Ramanathan, and S. K. Sankaranarayanan, Phys. Rev. E 88, 062119 (2013).

[18] G. Trefalt, Phys. Rev. E 93, 032612 (2016).

[19] I. Langmuir, J. Chem. Phys. 6, 873 (1938).

[20] W. A. Ducker, T. J. Senden, and R. M. Pashley, Langmuir 8, 1831 (1992).

[21] H. J. Butt, B. Cappella, and M. Kappl, Surf. Sci. Rep. 59, 1 (2005).

[22] V. Valmacco, M. Elzbieciak-Wodka, C. Besnard, P. Maroni, G. Trefalt, and M. Borkovec, Nanoscale Horiz. 1, 325 (2016).

[23] M. Porus, P. Maroni, and M. Borkovec, Langmuir 28, 5642 (2012).

[24] G. S. Manning, J. Chem. Phys. 51, 924 (1969).

[25] A. J. Milling, J. Phys. Chem. 100, 8986 (1996).

[26] S. Biggs, D. C. Prieve, and R. R. Dagastine, Langmuir 21, 5421 (2005).

[27] A. Takahashi, N. Kato, and M. Nagasawa, J. Phys. Chem. 74, 944 (1970).

[28] C. Wandrey, D. Hunkeler, U. Wendler, and W. Jaeger, Macromolecules 33, 7136 (2000).

[29] J. Yashiro and T. Norisuye, J. Polym. Sci. B 40, 2728 (2002).

[30] E. Trizac and G. Téllez, Phys. Rev. Lett. 96, 038302 (2006).

[31] M. Brunner, J. Dobnikar, H. H. von Grunberg, and C. Bechinger, Phys. Rev. Lett. 92, 078301 (2004). 